Discrete Comput Geom 33:27-41 (2005)

DOI: $10.1007 / \mathrm{s} 00454-004-1100-\mathrm{z}$

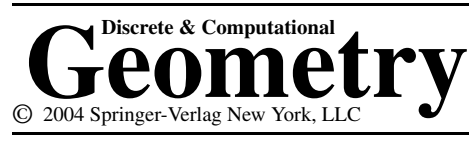

\title{
Some Low Distortion Metric Ramsey Problems*
}

\author{
Yair Bartal, ${ }^{1}$ Nathan Linial, ${ }^{1}$ Manor Mendel,${ }^{1}$ and Assaf Naor ${ }^{2}$ \\ ${ }^{1}$ Institute of Computer Science, Hebrew University, \\ Jerusalem 91904, Israel \\ \{yair,nati,mendelma\}@cs.huji.ac.il \\ ${ }^{2}$ Theory Group, Microsoft Research, \\ One Microsoft Way 113/2131, Redmond, WA 98052-6399, USA \\ anaor@microsoft.com
}

\begin{abstract}
In this note we consider the metric Ramsey problem for the normed spaces $\ell_{p}$. Namely, given some $1 \leq p \leq \infty$ and $\alpha \geq 1$, and an integer $n$, we ask for the largest $m$ such that every $n$-point metric space contains an $m$-point subspace which embeds into $\ell_{p}$ with distortion at most $\alpha$. In [1] it is shown that in the case of $\ell_{2}$, the dependence of $m$ on $\alpha$ undergoes a phase transition at $\alpha=2$. Here we consider this problem for other $\ell_{p}$, and specifically the occurrence of a phase transition for $p \neq 2$. It is shown that a phase transition does occur at $\alpha=2$ for every $p \in[1,2]$. For $p>2$ we are unable to determine the answer, but estimates are provided for the possible location of such a phase transition. We also study the analogous problem for isometric embedding and show that for every $1<p<\infty$ there are arbitrarily large metric spaces, no four points of which embed isometrically in $\ell_{p}$.
\end{abstract}

\section{Introduction}

A Ramsey-type theorem states that large systems necessarily contain large, highly structured subsystems. Here we consider Ramsey-type problems for finite metric spaces, interpreting "highly structured" as having low distortion embedding in $\ell_{p}$.

A mapping between two metric spaces $f: M \rightarrow X$ is called an embedding of $M$ in $X$. The distortion of the embedding is defined as

$$
\operatorname{dist}(f)=\sup _{\substack{x, y \in M \\ x \neq y}} \frac{d_{X}(f(x), f(y))}{d_{M}(x, y)} \cdot \sup _{\substack{x, y \in M \\ x \neq y}} \frac{d_{M}(x, y)}{d_{X}(f(x), f(y))} .
$$

\footnotetext{
* The first two authors were supported in part by a grant from the Israeli National Science Foundation, and the third author was supported in part by the Landau Center.
} 
The least distortion required to embed $M$ in $X$ is denoted by $c_{X}(M)$. When $c_{X}(M) \leq \alpha$ we say that $M \alpha$-embeds in $X$. In this note we study the following notion.

Definition 1 (Metric Ramsey Function). We denote by $R_{X}(\alpha, n)$ the largest integer $m$ such that every $n$-point metric space has a subspace of size $m$ that $\alpha$-embeds into $X$.

When $X=\ell_{p}$ we use the notations $c_{p}$ and $R_{p}$. Note that for $p \in[1, \infty]$, it is always true that $R_{p}(\alpha, n) \geq R_{2}(\alpha, n)$. When $\alpha=1$ we drop it from the notation, i.e., $R_{X}(n)=R_{X}(1, n)$.

Bourgain et al. [4] study this function for $X=\ell_{2}$, as a metric analog of Dvoretzky's theorem [7]. They prove:

Theorem 1 [4]. For any $\alpha>1$ there exists $C(\alpha)>0$ such that $R_{2}(\alpha, n) \geq C(\alpha) \log n$. Furthermore, there exists $\alpha_{0}>1$ such that $R_{2}\left(\alpha_{0}, n\right)=O(\log n)$.

In [1] the metric Ramsey problem is studied comprehensively. In particular, the following phase transition is established in the case of $X=\ell_{2}$.

Theorem 2 [1]. Let $n \in \mathbb{N}$. Then:

1. For every $1<\alpha<2: c(\alpha) \log n \leq R_{2}(\alpha, n) \leq 2 \log n+C(\alpha)$, where $c(\alpha)$ and $C(\alpha)$ may depend only on $\alpha$.

2. For every $\alpha>2$ : $n^{c^{\prime}(\alpha)} \leq R_{2}(\alpha, n) \leq n^{C^{\prime}(\alpha)}$, where $c^{\prime}(\alpha)$ and $C^{\prime}(\alpha)$ depend only on $\alpha$ and $0<c^{\prime}(\alpha) \leq C^{\prime}(\alpha)<1$. Moreover, $c^{\prime}(\alpha)$ tends to 1 as $\alpha$ tends to $\infty$.

By Dvoretzky's theorem, the lower bound in part 2 of Theorem 2 implies in particular that if $\alpha>2$, and $X$ is any infinite-dimensional normed space, then $R_{X}(\alpha, n) \geq n^{c^{\prime}(\alpha)}$. Therefore, in our search for a possible phase transition for $R_{p}(\cdot, n), p \neq 2$, it is natural to extend the upper bound in part 1 of Theorem 2 to this range. The main result proved in this note is the following:

Theorem 3. There is an absolute constant $c>0$ such that for every $0<\delta<1$ :

1. For $1 \leq p<2, R_{p}(2-\delta, n) \leq e^{c / \delta^{2}} \log n$.

2. For $2<p<\infty, R_{p}\left(2^{2 / p}-\delta, n\right) \leq e^{c / p^{2} \delta^{2}} \log n$.

Thus we extend the result of [1] to show that a phase transition occurs in the metric Ramsey problem for $\ell_{p}, p \in[1,2)$, at $\alpha=2$. The asymptotic behavior of $R_{p}(\alpha, n)$ for $p>2$, and $\alpha \in\left[2^{2 / p}, 2\right]$, is left as an open problem. In particular, we do not know whether or not this function undergoes a similar phase transition. We find this problem potentially significant: if there is a phase transition at 2 also in the range $2<p<\infty$, then this result will certainly be of great interest. On the other hand, if it is possible to improve the lower bound in part 2 of Theorem 2 for $p>2$ and certain distortions strictly less than 2 , then this would involve an embedding technique that is different from the method used in [1], which does not distinguish between the various $\ell_{p}$ spaces.

The proof of the upper bound on $R_{2}(\alpha, n)$ for $\alpha<2$ stated in Theorem 2 uses the Johnson-Lindenstrauss dimension reduction lemma for $\ell_{2}$ [10]. For $\ell_{p}, p \neq 2$, no 
such dimension reduction is known to hold. (Recent work [5], [11] shows that dimension reduction does not, in general, hold in $\ell_{1}$.) Our proof is based on a non-trivial modification of the random construction in [4], in the spirit of Erdös' upper bound on the Ramsey numbers [9], [3]. In the process we prove tight bounds on the embeddability of the metrics of complete bipartite graphs in $\ell_{p}$. Specifically we show that

$$
c_{p}\left(K_{n, n}\right)= \begin{cases}2-\Theta\left(n^{-1}\right), & p \in[1,2], \\ 2^{2 / p}-\Theta\left((p n)^{-1}\right), & p>2 .\end{cases}
$$

The second part of this note addresses the isometric Ramsey problem for $p \in(1, \infty)$. It turns out that this problem is naturally tackled within the class of uniformly convex normed spaces (see Section 3 for the definition).

Theorem 4 (Isometric Ramsey Problem). Let $X$ be a uniformly convex normed space with $\operatorname{dim}(X) \geq 2$. Then $R_{X}(1, n)=3$ for $n \geq 3$.

Since $\ell_{p}$ is uniformly convex for $p \in(1, \infty)$, the conclusion of Theorem 4 holds in these cases. Note that the theorem does not apply for $\ell_{1}$ and $\ell_{\infty}$ which are not uniformly convex. Specifically, it is known that $\ell_{\infty}$ is universal in that it contains an isometric copy of every finite metric space, whence $R_{\infty}(n)=n$. It is known [6] that any four-point metric space is isometrically embeddable in $\ell_{1}$, and therefore $R_{1}(n) \geq 4$ for $n \geq 4$. The determination of $R_{1}(n)$ is left as an open problem.

\section{An Upper Bound for $\alpha<2$}

In this section we prove that for any $\alpha<\min \left\{2,2^{2 / p}\right\}, R_{p}(\alpha, n)=O(\log n)$. Our technique both improves and simplifies the technique of [4], which is itself in the spirit of Erdös' original upper bound for the Ramsey coloring numbers. The basic idea is to exploit a universality property of random graphs $G \in G\left(n, \frac{1}{2}\right)$. Namely, that any fixed graph of constant size appears as an induced subgraph of every induced subgraph of $G$ of size $\Omega(\log n)$. More precisely, we define the following notion of universality.

Definition 2. Let $H$ be a graph. A graph $G$ is called $(H, s)$-universal if every set of $s$ vertices in $G$ contains an induced subgraph isomorphic to $H$.

Proposition 1. For every $k$-vertex graph $H$ there exists a constant $C>0$ and an integer $n_{0}$ such that for any $n>n_{0}$ there exists an $(H, C \log n)$-universal graph on $n$ vertices. Furthermore,

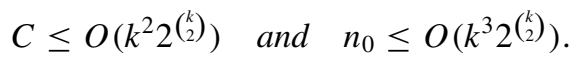

Such facts are well known in random graph theory, and similar arguments can be found for example in [13]. We sketch the standard details for the sake of completeness.

Recall that a family of sets $\mathcal{F}$ is called almost disjoint if $|A \cap B| \leq 1$ for every $A, B \in \mathcal{F}$. In what follows, given a set $S$ and an integer $k$, we denote by $\left(\begin{array}{l}S \\ k\end{array}\right)$ the set of all $k$-point subsets of $S$. 
Lemma 2. For every integer $k$ and a finite set $S$ of cardinality $s=|S|>2 k^{2}$, there exists an almost disjoint family $K \subset\left(\begin{array}{l}S \\ k\end{array}\right)$, such that $|K| \geq\lfloor s / 2 k\rfloor^{2}$.

Proof. Let $p$ be a prime satisfying $s / 2 k \leq p \leq s / k$, and assume that

$$
L=\left\{(i, j) ; i, j \in \mathbb{Z}_{p}, i \in\{0, \ldots, k-1\}\right\} \subseteq S .
$$

For each $a, b \in \mathbb{Z}_{p}$ (the field of residues modulo $p$ ), define

$$
A_{a, b}=\{(i, j) ; j \equiv a i+b(\bmod p), i \in\{0, \ldots, k-1\}\},
$$

and take $K=\left\{A_{a, b} \mid a, b \in \mathbb{Z}_{p}\right\}$. The set $K$ is easily checked to satisfy the requirements.

As usual $G\left(n, \frac{1}{2}\right)$ denotes the model of random graphs in which each edge on $n$ vertices is chosen independently with probability $\frac{1}{2}$.

Lemma 3. Let $H$ be a $k$-vertex graph and let $s>2 k^{2}$. The probability that a random graph $G \in G\left(s, \frac{1}{2}\right)$ does not contain an induced subgraph isomorphic to $H$, is at most

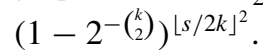

Proof. Construct, as in Lemma 2, an almost disjoint family $\mathcal{F}$ of $\lfloor s / 2 k\rfloor^{2}$ subsets of $\{1, \ldots, s\}$, the vertex set of $G$. If $F_{1} \neq F_{2} \in \mathcal{F}$, then the event that the restriction of $G$ to $F_{1}$ (resp. $F_{2}$ ) is isomorphic to $H$ is independent. Hence, the probability that none of the sets $F \in \mathcal{F}$ spans a subgraph isomorphic to $H$ is at most $\left(1-2^{-\left(\begin{array}{c}k \\ 2\end{array}\right)}\right)^{\lfloor s / 2 k\rfloor^{2}}$.

Proof of Proposition 1. Let $G$ be a random graph in $G\left(n, \frac{1}{2}\right)$. By the previous lemma, the expected number of sets of $s$ vertices which contain no induced isomorphic copy of

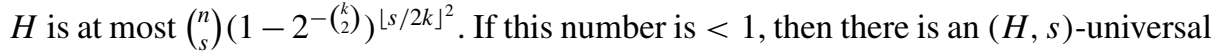
graph, as claimed. It is an easy matter to check that this holds with the parameters as stated.

A class $\mathcal{C}$ of finite metric spaces is called a metric class if it is closed under isometries. $\mathcal{C}$ is said to be hereditary if $M \in \mathcal{C}$ and $N \subset M$ imply $N \in \mathcal{C}$. We call a metric space $(X, d)$ a $\{0,1,2\}$ metric space if for all $x, y \in X, d(x, y) \in\{0,1,2\}$. There is a simple $1: 1$ correspondence between graphs and $\{0,1,2\}$ metrics. Namely, associated with a $\{0,1,2\}$ metric space $M=(X, d)$ is the graph $G=(X, E)$ where $\{x, y\} \in E$ iff $d_{M}(x, y)=1$.

Lemma 4. Let $\mathcal{C}$ be a hereditary metric class of finite metric spaces, and suppose that there exists some finite $\{0,1,2\}$ metric space $M_{0}$ which is not in $P$. Then there exist metric spaces $M=M_{n}$ of arbitrarily large size $n$ such that every subspace $S \subset M_{n}$ with at least $C \log n$ points is not in $\mathcal{C}$. The constant $C$ depends only on the cardinality of $M_{0}$.

Proof. Let $H_{0}$ be the graph corresponding to the metric space $M_{0}$. We apply Proposition 1, to construct arbitrarily large graphs $G_{n}=\left(V_{n}, E_{n}\right)$ with $\left|V_{n}\right|=n$, in which every 
set of $\geq C \log n$ vertices contains an induced subgraph isomorphic to $H_{0}$. Let $M_{n}$ be the $n$-point metric space corresponding to $G_{n}$. It follows that every subspace of $M_{n}$ of size $\geq C \log n$ contains a metric subspace that is isometric to $M_{0}$. Since $\mathcal{C}$ is hereditary, $S \notin \mathcal{C}$.

Note that $\left\{M ; M\right.$ is a metric space, $\left.c_{p}(M) \leq \alpha\right\}$ is a hereditary metric class. Therefore, in order to show that for $\alpha<2, R_{p}(\alpha, n)=O(\log n)$, it is enough to find a $\{0,1,2\}$ metric space whose $\ell_{p}$ distortion is greater than $\alpha$. We use the complete bipartite graphs $K_{n, n}$. The $\ell_{p}$-distortion of $K_{n, n}, 1 \leq p<\infty$, is estimated in the following proposition.

Proposition 5. For every $1 \leq p \leq 2$,

$$
2\left(\frac{n-1}{n}\right)^{1 / p} \leq c_{p}\left(K_{n, n}\right) \leq 2 \sqrt{\frac{n-1}{n}} .
$$

For every $2 \leq p<\infty$

$$
2^{2 / p}\left(\frac{n-1}{n}\right)^{1 / p} \leq c_{p}\left(K_{n, n}\right) \leq 2^{2 / p}\left(1-\frac{1}{2 n}\right)^{1 / p} .
$$

Before proving Proposition 5, we deduce the main result of this section:

Theorem 5. There is an absolute constant $c>0$ such that for every $0<\delta<1$, if $1 \leq p \leq 2$, then

$$
R_{p}(2-\delta, n) \leq e^{c / \delta^{2}} \log n,
$$

and if $2<p<\infty$, then

$$
R_{p}\left(2^{2 / p}-\delta, n\right) \leq e^{c / p^{2} \delta^{2}} \log n .
$$

Proof. Proposition 1 implies that there is an absolute constant $C$ such that for every $n \geq 2^{C k^{3}}$ there exists a $\{0,1,2\}$ metric space $M_{n}$ such that any subset $S \subset M_{n}$ of cardinality at least $2^{C k^{2}} \log n$ contains an isometric copy of $K_{k, k}$.

We start with $1 \leq p \leq 2$. Let $k=\lfloor 2 / \delta\rfloor+1$. By Proposition 5,

$$
c_{p}\left(K_{k, k}\right) \geq 2\left(1-\frac{1}{k}\right)^{1 / p}>2\left(1-\frac{\delta}{2}\right)=2-\delta,
$$

so that for $n$ large enough ( $\geq e^{C^{\prime} / \delta^{3}}$ ), and hence for all $n$ (by proper choice of constants),

$$
R_{p}(2-\delta, n) \leq e^{C^{\prime} / \delta^{2}} \log n .
$$

When $p>2$ take $k=2\lfloor 4 / p \delta\rfloor$. In this case one easily verifies that

$$
c_{p}\left(K_{k, k}\right) \geq 2^{2 / p}\left(1-\frac{1}{k}\right)^{1 / p} \geq 2^{2 / p}-\delta,
$$

from which the required result follows as above. 
In order to prove Proposition 5, we need some preparation.

Lemma 6. Let $A=\left(a_{i j}\right)$ be an $n \times n$ matrix and $2 \leq p<\infty$. Then

$$
\sum_{i=1}^{n} \sum_{j=1}^{n}\left(\left|\sum_{k=1}^{n} a_{i k}-\sum_{k=1}^{n} a_{j k}\right|^{p}+\left|\sum_{k=1}^{n} a_{k i}-\sum_{k=1}^{n} a_{k j}\right|^{p}\right) \leq \frac{(2 n)^{p}}{2} \sum_{i=1}^{n} \sum_{j=1}^{n}\left|a_{i j}\right|^{p} .
$$

Proof. We identify $\ell_{p}^{n^{2}}$ with the space of all $n \times n$ matrices $A=\left(a_{i j}\right)$, equipped with the $\ell_{p}$ norm:

$$
\|A\|_{p}=\left(\sum_{i=1}^{n} \sum_{j=1}^{n}\left|a_{i j}\right|^{p}\right)^{1 / p}
$$

Define a linear operator $T: \mathbb{R}^{n^{2}} \rightarrow \mathbb{R}^{n^{2}} \oplus \mathbb{R}^{n^{2}}$ by

$$
T\left(a_{i j}\right)=\left(\sum_{k=1}^{n} a_{i k}-\sum_{k=1}^{n} a_{j k}\right)_{i j} \oplus\left(\sum_{k=1}^{n} a_{k i}-\sum_{k=1}^{n} a_{k j}\right)_{i j}
$$

For $q \geq 1$ denote $\|T\|_{q \rightarrow q}=\max _{A \neq 0}\|T(A)\|_{q} /\|A\|_{q}$. Our goal is to show that $\|T\|_{p \rightarrow p} \leq 2^{1-1 / p} n$. By a result from the complex interpolation theory for linear operators (see [2]), for $2 \leq p \leq \infty,\|T\|_{p \rightarrow p} \leq\|T\|_{2 \rightarrow 2}^{2 / p} \cdot\|T\|_{\infty \rightarrow \infty}^{1-2 / p}$. It is therefore enough to prove the required estimate for $p=2$ and $p=\infty$. The case $p=\infty$ is simple:

$$
\|T(A)\|_{\infty}=\max _{1 \leq i, j \leq n} \max \left\{\left|\sum_{k=1}^{n} a_{i k}-\sum_{k=1}^{n} a_{j k}\right|,\left|\sum_{k=1}^{n} a_{k i}-\sum_{k=1}^{n} a_{k j}\right|\right\} \leq 2 n\|A\|_{\infty} .
$$

For $p=2$ we have to show that

$$
\sum_{i=1}^{n} \sum_{j=1}^{n}\left(\left|\sum_{k=1}^{n} a_{i k}-\sum_{k=1}^{n} a_{j k}\right|^{2}+\left|\sum_{k=1}^{n} a_{k i}-\sum_{k=1}^{n} a_{k j}\right|^{2}\right) \leq 2 n^{2} \sum_{i=1}^{n} \sum_{j=1}^{n}\left|a_{i j}\right|^{2} .
$$

This inequality follows from the following elementary identity:

$$
\begin{aligned}
2 n^{2} \sum_{i=1}^{n} \sum_{j=1}^{n} a_{i j}^{2}= & \sum_{i=1}^{n} \sum_{j=1}^{n}\left[\left(\sum_{k=1}^{n} a_{i k}-\sum_{k=1}^{n} a_{j k}\right)^{2}+\left(\sum_{k=1}^{n} a_{k i}-\sum_{k=1}^{n} a_{k j}\right)^{2}\right] \\
& +2 \sum_{i=1}^{n} \sum_{j=1}^{n}\left(n a_{i j}-\sum_{k=1}^{n} a_{i k}-\sum_{k=1}^{n} a_{k j}\right)^{2} .
\end{aligned}
$$

Corollary 7. Let $1 \leq p<\infty$ and $x_{1}, \ldots, x_{n}, y_{1}, \ldots, y_{n} \in \ell_{p}$. Then if $2 \leq p<\infty$,

$$
\sum_{i=1}^{n} \sum_{j=1}^{n}\left(\left\|x_{i}-x_{j}\right\|_{p}^{p}+\left\|y_{i}-y_{j}\right\|_{p}^{p}\right) \leq 2^{p-1} \sum_{i=1}^{n} \sum_{j=1}^{n}\left\|x_{i}-y_{j}\right\|_{p}^{p} .
$$


If $1 \leq p \leq 2$, then

$$
\sum_{i=1}^{n} \sum_{j=1}^{n}\left(\left\|x_{i}-x_{j}\right\|_{p}^{p}+\left\|y_{i}-y_{j}\right\|_{p}^{p}\right) \leq 2 \sum_{i=1}^{n} \sum_{j=1}^{n}\left\|x_{i}-y_{j}\right\|_{p}^{p} .
$$

Proof. By summation it is clearly enough to prove these inequalities for $x_{1}, \ldots, x_{n}$, $y_{1}, \ldots, y_{n} \in \mathbb{R}$. If $2 \leq p<\infty$, then the required result follows from an application of Lemma 6 to the matrix $a_{i j}=x_{i}-y_{j}$. If $1 \leq p \leq 2$, then consider $\ell_{p}$ equipped with the metric $d(x, y)=\|x-y\|_{p}^{p / 2}$. It is well known (see [14]) that $\left(\ell_{p}, d\right)$ embeds isometrically in $\ell_{2}$, so that the case $1 \leq p \leq 2$ follows from the case $p=2$.

Remark. In [8] Enflo defined the notion on generalized roundness of a metric space. A metric space $(M, d)$ is said to have generalized roundness $q \geq 0$ if for every $x_{1}, \ldots, x_{n}, y_{1}, \ldots, y_{n} \in M$,

$$
\sum_{i=1}^{n} \sum_{j=1}^{n}\left(d\left(x_{i}, x_{j}\right)^{q}+d\left(y_{i}, y_{j}\right)^{q}\right) \leq 2 \sum_{i=1}^{n} \sum_{j=1}^{n} d\left(x_{i}, y_{j}\right)^{q} .
$$

Enflo proved that Hilbert space has generalized roundness 2 and in [12] the concept of generalized roundness was investigated and was shown to be equivalent to the notion of negative type (see [6] and [14] for the definition). Particularly, it was proved in [12] that for $1 \leq p<2, \ell_{p}$ has generalized roundness $p$, which is precisely the second statement in Corollary 7. For the case $p=1$, simpler more direct proofs can be given which do not use reduction to the case $p=2$, see, e.g., [6]. Observe that Lemma 6 would follow simply by convexity had it not been for the additional factor $\frac{1}{2}$ on the right-hand side. This factor is crucial for our purposes, and this is why the interpolation argument was needed.

Proof of Proposition 5. We identify $K_{n, n}$ with the metric on $\left\{u_{1}, \ldots, u_{n}, v_{1}, \ldots, v_{n}\right\}$ where $d\left(u_{i}, u_{j}\right)=d\left(v_{i}, v_{j}\right)=2$ for all $i \neq j$, and $d\left(u_{i}, v_{j}\right)=1$ for every $1 \leq i, j \leq n$. Fix some $1 \leq p<\infty$ and let $f:\left\{u_{1}, \ldots, u_{n}, v_{1}, \ldots, v_{n}\right\} \rightarrow \ell_{p}$ be an embedding such that for every $x, y \in K_{n, n}, d(x, y) \leq\|f(x)-f(y)\|_{p} \leq L d(x, y)$. Then

$$
\sum_{i=1}^{n} \sum_{j=1}^{n}\left(\left\|f\left(u_{i}\right)-f\left(u_{j}\right)\right\|_{p}^{p}+\left\|f\left(v_{i}\right)-f\left(v_{j}\right)\right\|_{p}^{p}\right) \geq 2 n(n-1) 2^{p}
$$

and

$$
\sum_{i=1}^{n} \sum_{j=1}^{n}\left\|f\left(u_{i}\right)-f\left(v_{j}\right)\right\|_{p}^{p} \leq n^{2} L^{p} .
$$

For $1 \leq p \leq 2$ Corollary 7 gives

$$
2 n(n-1)^{p} 2^{p} \leq 2 n^{2} L^{p} \quad \Longrightarrow \quad L \geq 2\left(\frac{n-1}{n}\right)^{1 / p} .
$$


For $2 \leq p<\infty$ we get that

$$
2 n(n-1) 2^{p} \leq 2^{p-1} n^{2} L^{p} \quad \Longrightarrow \quad L \geq 2^{2 / p}\left(\frac{n-1}{n}\right)^{1 / p} .
$$

This proves the required lower bounds on $c_{p}\left(K_{n, n}\right)$.

To prove the upper bound assume first that $p=2$ and denote by $\left\{e_{i}\right\}_{i=1}^{\infty}$ the standard unit vectors in $\ell_{2}$. Define $f: K_{n, n} \rightarrow \ell_{2}^{2 n}$ by

$$
\begin{aligned}
& f\left(u_{i}\right)=\sqrt{2}\left(e_{i}-\frac{1}{n} \sum_{j=1}^{n} e_{j}\right), \\
& f\left(v_{i}\right)=\sqrt{2}\left(e_{n+i}-\frac{1}{n} \sum_{j=1}^{n} e_{n+j}\right) .
\end{aligned}
$$

Then for $i \neq j,\left\|f\left(u_{i}\right)-f\left(u_{j}\right)\right\|_{2}=\left\|f\left(v_{i}\right)-f\left(v_{j}\right)\right\|_{2}=2=d\left(u_{i}, u_{j}\right)=d\left(v_{i}, v_{j}\right)$. On the other hand,

$$
\begin{aligned}
\left\|f\left(u_{i}\right)-f\left(v_{j}\right)\right\|_{2} & =\sqrt{\left\|f\left(u_{i}\right)\right\|_{2}^{2}+\left\|f\left(v_{j}\right)\right\|_{2}^{2}} \\
& =\sqrt{4\left(1-\frac{1}{n}\right)^{2}+4(n-1) \cdot \frac{1}{n^{2}}}=2 \sqrt{\frac{n-1}{n}}
\end{aligned}
$$

This finishes the calculation of $c_{2}\left(K_{n, n}\right)$. For $1 \leq p<2$, since for every $\varepsilon>0$ and for every $k, \ell_{p}$ contains a $(1+\varepsilon)$ distorted copy of $\ell_{2}^{k}$, we get the estimate $c_{p}\left(K_{n, n}\right) \leq$ $2 \sqrt{(n-1) / n}$.

The case $2<p<\infty$ requires a different embedding. We begin by describing an embedding with distortion $2^{2 / p}$ and then explain how to modify it so as to reduce the distortion by a factor of $(1-1 / 2 n)^{1 / p}$. Let $z_{1}, \ldots, z_{n}$ be a collection of $n$ mutually orthogonal \pm 1 vectors of dimension $m=O(n)$. (For example, the first $n$ rows in an $m \times m$ Hadamard matrix.) In our first embedding we define $f\left(u_{i}\right)$ as the $(2 m)$-dimensional vector $\left(z_{i}, 0\right)$, namely, $z_{i}$ concatenated with $m$ zeros. Likewise, $f\left(v_{i}\right)=\left(0, z_{i}\right)$ for all $i$. Now $\left\|f\left(u_{i}\right)-f\left(u_{j}\right)\right\|_{p}=2(m / 2)^{1 / p}$ and $\left\|f\left(u_{i}\right)-f\left(v_{j}\right)\right\|_{p}=(2 m)^{1 / p}$, and so $f$ has distortion $2^{2 / p}$. To get the $(1-1 / 2 n)^{1 / p}$ improvement, note that for some $m \leq 4 n$ it is possible to select the $z_{i}$ so that the $m$ th coordinate in all of them is +1 . Modify the previous construction to an embedding into $2 m-1$ dimensions as follows: now $g\left(u_{i}\right)$ is $z_{i}$ concatenated with $m-1$ zeros, whereas $g\left(v_{i}\right)$ has zeros in the first $m-1$ coordinates, 1 in the $m$ th and this is followed by the first $m-1$ coordinates of the vector $z_{i}$. The easy details are omitted.

Remark. The upper bounds in Proposition 5 were not used in the proof of Theorem 5. Apart from their intrinsic interest, these upper estimates show that the above technique cannot prove an upper bound of $O(\log n)$ on $R_{2}(2-\varepsilon, n)$ which is independent of $\varepsilon$. In fact, this can never be achieved using $\{0,1,2\}$ metric spaces due to the following proposition.

Proposition 8. Let X be an n-point $\{0,1,2\}$ metric space. Then $c_{2}(X) \leq 2 \sqrt{(n-1) / n}$. 
Proof. We think of $X$ as a metric on $\{1, \ldots, n\}$ and denote $d(i, j)=d_{i j}$. Define an $n \times n$ matrix $A=\left(a_{i j}\right)$ as follows:

$$
a_{i j}=\left\{\begin{array}{lll}
2 & \text { if } \quad i=j, \\
0 & \text { if } \quad d_{i j}=2, \\
\frac{2}{n} & \text { if } \quad d_{i j}=1 .
\end{array}\right.
$$

We claim that $A$ is positive semidefinite. Indeed, for any $z \in \mathbb{R}^{n}$,

$$
\begin{aligned}
\langle A z, z\rangle & =\sum_{i=1}^{n} \sum_{j=1}^{n} a_{i j} z_{i} z_{j} \\
& \geq \sum_{i=1}^{n} 2 z_{i}^{2}-\sum_{i \neq j} \frac{2}{n}\left|z_{i}\right| \cdot\left|z_{j}\right| \\
& \geq \sum_{i=1}^{n} 2 z_{i}^{2}-\sum_{i=1}^{n} \sum_{j=1}^{n} \frac{2}{n}\left|z_{i}\right| \cdot\left|z_{j}\right| \\
& =2\|z\|_{2}^{2}-\frac{2}{n}\|z\|_{1}^{2} \geq 2\|z\|_{2}^{2}-\frac{2}{n} n\|z\|_{2}^{2}=0 .
\end{aligned}
$$

In particular, it follows that $A$ has a square root, denoted $A^{1 / 2}$. Let $e_{1}, \ldots, e_{n}$ be the standard unit vectors in $\mathbb{R}^{n}$. Define $f: X \rightarrow \mathbb{R}^{n}$ by $f(i)=A^{1 / 2} e_{i}$. Now,

$$
\|f(i)-f(j)\|_{2}^{2}=\left\langle A e_{i}, e_{i}\right\rangle+\left\langle A e_{j}, e_{j}\right\rangle-2\left\langle A e_{i}, e_{j}\right\rangle=a_{i i}+a_{j j}-2 a_{i j},
$$

so that if $d_{i j}=1$, then $\|f(i)-f(j)\|_{2}=\sqrt{4-4 / n}$ and if $d_{i j}=2$, then $\| f(i)-$ $f(j) \|_{2}=2$. It follows that

$$
\operatorname{dist}(f)=2 \sqrt{\frac{n-1}{n}}
$$

\section{The Isometric Ramsey Problem}

In this section we prove that for $n \geq 3,1<p<\infty, R_{p}(n)=R_{p}(1, n)=3$. In fact, we show that this is true for any uniformly convex normed space. We begin by sketching an argument that is specific to $\ell_{2}$ :

Proposition 9. $R_{2}(n)=3$ for $n \geq 3$.

Proof. That $R_{2}(n) \geq 3$ follows since any metric space on three points embeds isometrically in $\ell_{2}^{2}$. To show that $R_{2}(n) \leq 3$, we construct a metric space on $n>3$ points, no fourpoint subspace of which embeds isometrically in $\ell_{2}$. Fix an integer $n>3$ and let $\left\{a_{i}\right\}_{i=0}^{n}$ be an increasing sequence such that $a_{0}=0, a_{1}=1$, and for $1 \leq i<n, a_{i+1} \geq 2(n+1) a_{i}$. Fix some $0<\varepsilon<1 /\left(2 a_{n}\right)$. It is easily verified that $d(i, j)=|i-j|-\varepsilon a_{|i-j|}$ is a metric on $\{1,2, \ldots, n\}$. We show that for $\varepsilon$ small enough no four points in $(\{1, \ldots, n\}, d) \mathrm{em}$ bed isometrically in $\ell_{2}$. Fix four integers $1 \leq i_{1}<i_{2}<i_{3}<i_{4} \leq n$ and set $j=i_{2}-i_{1}$, 
$k=i_{3}-i_{2}, l=i_{4}-i_{3}$. Suppose that for every $\varepsilon>0$ there exists an isometric embedding $f:\left(\left\{i_{1}, i_{2}, i_{3}, i_{4}\right\}, d\right) \rightarrow \ell_{2}^{3}$. Without loss of generality we may assume that $f\left(i_{1}\right)=(\alpha, \beta, \gamma), f\left(i_{2}\right)=(0,0,0), f\left(i_{3}\right)=\left(k-\varepsilon a_{k}, 0,0\right)$, and $f\left(i_{4}\right)=(p, q, 0)$.

Then

$$
\begin{aligned}
2 \alpha\left(k-\varepsilon a_{k}\right) & =2\left\langle f\left(i_{1}\right), f\left(i_{3}\right)\right\rangle \\
& =\left\|f\left(i_{1}\right)-f\left(i_{2}\right)\right\|_{2}^{2}+\left\|f\left(i_{3}\right)-f\left(i_{2}\right)\right\|_{2}^{2}-\left\|f\left(i_{3}\right)-f\left(i_{1}\right)\right\|_{2}^{2} \\
& =\left(j-\varepsilon a_{j}\right)^{2}+\left(k-\varepsilon a_{k}\right)^{2}-\left(j+k-\varepsilon a_{j+k}\right)^{2} .
\end{aligned}
$$

Hence,

$$
\alpha \leq-j+\frac{\varepsilon}{k}\left[(k+j) a_{k+j}-j a_{j}-k a_{k}-j a_{k}\right]+O\left(\varepsilon^{2}\right) .
$$

Similarly,

$$
p \geq(k+l)+\frac{\varepsilon}{k}\left[(k+l) a_{k}-(k+l) a_{k+l}-k a_{k}+l a_{l}\right]+O\left(\varepsilon^{2}\right) .
$$

Now

$$
\begin{aligned}
j+k+l- & \varepsilon a_{j+k+l} \\
= & \left\|f\left(i_{4}\right)-f\left(i_{1}\right)\right\|_{2} \\
\geq & p-\alpha \\
\geq & j+k+l \\
& +\frac{\varepsilon}{k}\left[(k+l) a_{k}-(k+l) a_{k+l}+l a_{l}-(k+j) a_{k+j}+j a_{j}+j a_{k}\right] \\
& +O\left(\varepsilon^{2}\right) .
\end{aligned}
$$

Letting $\varepsilon$ tend to zero we deduce that

$$
\begin{aligned}
a_{j+k+l} & \leq\left(1+\frac{j}{k}\right) a_{k+j}+\left(1+\frac{l}{k}\right) a_{k+l}-\frac{l}{k} a_{l}-\frac{j}{k} a_{j}-\frac{j+k+l}{k} a_{k} \\
& <2(n+1) a_{j+k+l-1},
\end{aligned}
$$

which is a contradiction.

The argument above is quite specific to $\ell_{2}$, and so we now consider any uniformly convex normed space. The modulus of uniform convexity of a normed space $X$ is defined by

$$
\delta_{X}(\varepsilon)=\inf \left\{1-\frac{\|a+b\|}{2} ;\|a\|,\|b\| \leq 1 \text { and }\|a-b\| \geq \varepsilon\right\} .
$$

$X$ is said to be uniformly convex if $\delta_{X}(\varepsilon)>0$ for every $0<\varepsilon \leq 2$. The $L_{p}$ spaces $1<p<\infty$ are known to be uniformly convex. For a uniformly convex space $X, \delta_{X}$ is known to be continuous and strictly increasing on $(0,2]$. 
Assume that $X$ is a uniformly convex normed space and $a, b \in X \backslash\{0\}$. Then

$$
\begin{aligned}
\left\|\frac{a}{\|a\|}+\frac{b}{\|b\|}\right\| & =\left\|\left(\frac{1}{\|a\|}+\frac{1}{\|b\|}\right)(a+b)-\frac{a}{\|b\|}-\frac{b}{\|a\|}\right\| \\
& \geq\left(\frac{1}{\|a\|}+\frac{1}{\|b\|}\right)\|a+b\|-\frac{\|a\|}{\|b\|}-\frac{\|b\|}{\|a\|} \\
& =2-\left(\frac{1}{\|a\|}+\frac{1}{\|b\|}\right)(\|a\|+\|b\|-\|a+b\|) .
\end{aligned}
$$

Now,

$$
\begin{aligned}
\delta_{X}\left(\left\|\frac{a}{\|a\|}-\frac{b}{\|b\|}\right\|\right) & \leq 1-\frac{1}{2} \cdot\left\|\frac{a}{\|a\|}+\frac{b}{\|b\|}\right\| \\
& \leq \frac{1}{2} \cdot\left(\frac{1}{\|a\|}+\frac{1}{\|b\|}\right)(\|a\|+\|b\|-\|a+b\|) .
\end{aligned}
$$

Hence

$$
\left\|\frac{a}{\|a\|}-\frac{b}{\|b\|}\right\| \leq \delta_{X}^{-1}\left(\frac{1}{2} \cdot\left(\frac{1}{\|a\|}+\frac{1}{\|b\|}\right)(\|a\|+\|b\|-\|a+b\|)\right) .
$$

Take $x, y, z \in X$ and apply this inequality for $a=x-y, b=y-z$. It follows that

$$
\begin{aligned}
\left\|y-\left(\frac{\|y-z\|}{\|x-y\|+\|y-z\|} \cdot x+\frac{\|x-y\|}{\|x-y\|+\|y-z\|} \cdot z\right)\right\| \\
\quad \leq \frac{\|x-y\| \cdot\|y-z\|}{\|x-y\|+\|y-z\|} \cdot \delta_{X}^{-1}\left(\frac{\|x-y\|+\|y-z\|-\|x-z\|}{\min \{\|x-y\|,\|y-z\|\}}\right) .
\end{aligned}
$$

This inequality is the way uniform convexity is going to be applied in the sequel. Indeed, we have the following "metric" consequence of it:

Lemma 10. Let $X$ be a uniformly convex normed space and let $x_{1}, x_{2}, x_{3}, x_{4} \in X$ be distinct. Then

$$
\begin{aligned}
\frac{\left\|x_{1}-x_{2}\right\|+}{\left\|x_{2}-x_{3}\right\|-\left\|x_{1}-x_{3}\right\|} & 2\left\|x_{2}-x_{3}\right\| \\
\leq & \delta_{X}^{-1}\left(\frac{\left\|x_{1}-x_{3}\right\|+\left\|x_{3}-x_{4}\right\|-\left\|x_{1}-x_{4}\right\|}{\min \left\{\left\|x_{1}-x_{3}\right\|,\left\|x_{3}-x_{4}\right\|\right\}}\right) \\
& \quad+\delta_{X}^{-1}\left(\frac{\left\|x_{2}-x_{3}\right\|+\left\|x_{3}-x_{4}\right\|-\left\|x_{2}-x_{4}\right\|}{\min \left\{\left\|x_{2}-x_{3}\right\|,\left\|x_{3}-x_{4}\right\|\right\}}\right) .
\end{aligned}
$$

Lemma 10 is a quantitative version of the fact that in a uniformly convex space, if $\left\|x_{1}-x_{4}\right\|$ is approximately $\left\|x_{1}-x_{3}\right\|+\left\|x_{3}-x_{4}\right\|$ and $\left\|x_{2}-x_{4}\right\|$ is approximately $\left\|x_{2}-x_{3}\right\|+\left\|x_{3}-x_{4}\right\|$, then $\left\|x_{1}-x_{3}\right\|$ is approximately $\left\|x_{1}-x_{2}\right\|+\left\|x_{2}-x_{3}\right\|$. This fact is geometrically evident since the first assumption implies that $x_{3}$ is almost on the line segment connecting $x_{1}$ and $x_{4}$ and $x_{2}$ is almost on the line segment connecting $x_{1}$ and $x_{3}$. It follows that $x_{2}$ is almost on the line segment connecting $x_{1}$ and $x_{3}$, as required. Since we are dealing with bi-Lipschitz embeddings, we must formulate this phenomenon without referring to "line segments." 
Proof of Lemma 10. Define

$$
\lambda=\frac{\left\|x_{3}-x_{4}\right\|}{\left\|x_{1}-x_{3}\right\|+\left\|x_{3}-x_{4}\right\|} \quad \text { and } \quad \mu=\frac{\left\|x_{3}-x_{4}\right\|}{\left\|x_{2}-x_{3}\right\|+\left\|x_{3}-x_{4}\right\|} .
$$

An application of (1) twice gives

$$
\begin{aligned}
\left\|x_{3}-\left(\lambda x_{1}+(1-\lambda) x_{4}\right)\right\| \leq & \frac{\left\|x_{1}-x_{3}\right\| \cdot\left\|x_{3}-x_{4}\right\|}{\left\|x_{1}-x_{3}\right\|+\left\|x_{3}-x_{4}\right\|} \\
& \cdot \delta_{X}^{-1}\left(\frac{\left\|x_{1}-x_{3}\right\|+\left\|x_{3}-x_{4}\right\|-\left\|x_{1}-x_{4}\right\|}{\min \left\{\left\|x_{1}-x_{3}\right\|,\left\|x_{3}-x_{4}\right\|\right\}}\right)
\end{aligned}
$$

and

$$
\begin{aligned}
\left\|x_{3}-\left(\mu x_{2}+(1-\mu) x_{4}\right)\right\| \leq & \frac{\left\|x-2-x_{3}\right\| \cdot\left\|x_{3}-x_{4}\right\|}{\left\|x_{2}-x_{3}\right\|+\left\|x_{3}-x_{4}\right\|} \\
& \cdot \delta_{X}^{-1}\left(\frac{\left\|x_{2}-x_{3}\right\|+\left\|x_{3}-x_{4}\right\|-\left\|x_{2}-x_{4}\right\|}{\min \left\{\left\|x_{2}-x_{3}\right\|,\left\|x_{3}-x_{4}\right\|\right\}}\right) .
\end{aligned}
$$

By symmetry, we may assume without loss of generality that $\lambda \leq \mu$. Now,

$$
\begin{aligned}
&\left\|x_{2}-\frac{\lambda(1-\mu)}{\mu(1-\lambda)} x_{1}-\frac{\mu-\lambda}{\mu(1-\lambda)} x_{3}\right\| \\
&=\frac{1}{\mu}\left\|\mu x_{2}+(1-\mu) x_{4}-x_{3}+\frac{1-\mu}{1-\lambda}\left(x_{3}-\lambda x_{1}-(1-\lambda) x_{4}\right)\right\| \\
& \leq \frac{1}{\mu}\left\|x_{3}-\mu x_{2}-(1-\mu) x_{4}\right\|+\frac{1-\mu}{\mu(1-\lambda)} \cdot\left\|x_{3}-\lambda x_{1}-(1-\lambda) x_{4}\right\| \\
& \leq \frac{\left\|x_{2}-x_{3}\right\|+\left\|x_{3}-x_{4}\right\|}{\left\|x_{3}-x_{4}\right\|} \cdot \frac{\left\|x_{2}-x_{3}\right\| \cdot\left\|x_{3}-x_{4}\right\|}{\left\|x_{2}-x_{3}\right\|+\left\|x_{3}-x_{4}\right\|} \\
& \cdot \delta_{X}^{-1}\left(\frac{\left\|x_{2}-x_{3}\right\|+\left\|x_{3}-x_{4}\right\|-\left\|x_{2}-x_{4}\right\|}{\min \left\{\left\|x_{2}-x_{3}\right\|,\left\|x_{3}-x_{4}\right\|\right\}}\right) \\
&+\frac{\left\|x_{2}-x_{3}\right\|}{\left\|x_{3}-x_{4}\right\|} \frac{\left\|x_{1}-x_{3}\right\|+\left\|x_{3}-x_{4}\right\|}{\left\|x_{1}-x_{3}\right\|}\left\|x_{3}\right\| \cdot\left\|x_{3}-x_{4}\right\| \\
& \cdot \delta_{X}^{-1}\left(\frac{\left\|x_{1}-x_{3}\right\|+\left\|x_{3}-x_{4}\right\|-\left\|x_{1}-x_{4}\right\|}{\min \left\{\left\|x_{1}-x_{3}\right\|,\left\|x_{3}-x_{4}\right\|\right\}}\right) \\
&=\left\|x_{2}-x_{3}\right\| \delta_{X}^{-1}\left(\frac{\left\|x_{1}-x_{3}\right\|+\left\|x_{3}-x_{4}\right\|-\left\|x_{1}-x_{4}\right\|}{\min \left\{\left\|x_{1}-x_{3}\right\|,\left\|x_{3}-x_{4}\right\|\right\}}\right) \\
&+\left\|x_{2}-x_{3}\right\| \delta_{X}^{-1}\left(\frac{\left\|x_{2}-x_{3}\right\|+\left\|x_{3}-x_{4}\right\|-\left\|x_{2}-x_{4}\right\|}{\min \left\{\left\|x_{2}-x_{3}\right\|,\left\|x_{3}-x_{4}\right\|\right\}}\right) .
\end{aligned}
$$

Additionally,

$$
\begin{aligned}
\left\|x_{2}-x_{1}\right\| \leq & \left\|x_{2}-\frac{\lambda(1-\mu)}{\mu(1-\lambda)} x_{1}-\frac{\mu-\lambda}{\mu(1-\lambda)} x_{3}\right\| \\
& +\left\|x_{1}-\frac{\lambda(1-\mu)}{\mu(1-\lambda)} x_{1}-\frac{\mu-\lambda}{\mu(1-\lambda)} x_{3}\right\| \\
= & \left\|x_{2}-\frac{\lambda(1-\mu)}{\mu(1-\lambda)} x_{1}-\frac{\mu-\lambda}{\mu(1-\lambda)} x_{3}\right\|+\frac{\mu-\lambda}{\mu(1-\lambda)}\left\|x_{1}-x_{3}\right\|
\end{aligned}
$$


and

$$
\begin{aligned}
\left\|x_{2}-x_{3}\right\| \leq & \left\|x_{2}-\frac{\lambda(1-\mu)}{\mu(1-\lambda)} x_{1}-\frac{\mu-\lambda}{\mu(1-\lambda)} x_{3}\right\| \\
& +\left\|x_{3}-\frac{\lambda(1-\mu)}{\mu(1-\lambda)} x_{1}-\frac{\mu-\lambda}{\mu(1-\lambda)} x_{3}\right\| \\
= & \left\|x_{2}-\frac{\lambda(1-\mu)}{\mu(1-\lambda)} x_{1}-\frac{\mu-\lambda}{\mu(1-\lambda)} x_{3}\right\|+\frac{\lambda(1-\mu)}{\mu(1-\lambda)}\left\|x_{1}-x_{3}\right\| .
\end{aligned}
$$

Summing up these estimates gives the required result.

We can now prove the main result of this section:

Theorem 6. Let $X$ be a uniformly convex normed space with $\operatorname{dim}(X) \geq 2$. Then for every $n \geq 3, R_{X}(n)=3$. Moreover, for every $\delta:(0,2] \rightarrow(0, \infty)$ which is continuous, increasing, and $\delta \leq \delta_{\ell_{2}}$, let $U C_{\delta}$ be the class of all normed spaces $X$ with $\delta_{X} \geq \delta$. Then for each $n \geq 3$ there is a constant $\varepsilon_{n}(\delta)>0$ such that $R_{U C_{\delta}}\left(1+\varepsilon_{n}(\delta), n\right)=3$.

The proof of Theorem 6 proceeds by constructing a space in which each quadruple violates the conclusion of Lemma 10. The construction is done iteratively, by adding one point at a time.

Proof of Theorem 6. That $R_{X}(n) \geq 3$ follows since any three-point metric embeds isometrically into any two-dimensional normed space, by a standard continuity argument.

Fix some $\delta:(0,2] \rightarrow(0, \infty)$ which is continuous, increasing, and $\delta \leq \delta_{\ell_{2}}$. We shall construct inductively a sequence $\left\{M_{n}\right\}_{n=3}^{\infty}$ of metric spaces and numbers $\left\{\eta_{n}\right\}_{n=3}^{\infty}$ such that:

(a) For every $n \geq 3, \eta_{n}>0$. Each $M_{n}$ is a metric on $\{1, \ldots, n\}$, and we denote $d_{i j}^{n}=d_{M_{n}}(i, j)$.

(b) For every $1 \leq i<j<k \leq n$,

$$
\begin{aligned}
d_{i, j}^{n}+d_{j k}^{n}- & d_{i, k}^{n}-\eta_{n} \\
& \geq 2 d_{j, k}^{n}\left[\delta^{-1}\left(\frac{d_{i, k}^{n}+d_{k, n}^{n}-d_{i, n}^{n}}{\min \left\{d_{i, k}^{n}, d_{k, n}^{n}\right\}}\right)+\delta^{-1}\left(\frac{d_{j, k}^{n}+d_{k, n}^{n}-d_{j, n}^{n}}{\min \left\{d_{j, k}^{n}, d_{k, n}^{n}\right\}}\right)\right] .
\end{aligned}
$$

Lemma 10 immediately implies that there is a constant $\varepsilon_{n}(\delta)>0$ such that for every $1 \leq i<j<k<l \leq n$ and for every normed space $X$ with $\delta_{X} \geq \delta$,

$$
c_{X}\left(\{i, j, k, l\}, d_{M_{n}}\right) \geq 1+\varepsilon_{n}(\delta),
$$

as required.

$M_{3}$ is the equilateral metric on $\{1,2,3\}$, in which case $\eta_{3}=1$. We construct $M_{n+1}=$ $\left(\{1, \ldots, n+1\}, d^{n+1}\right)$ as an extension of $M_{n}$, by setting

$$
d_{n, n+1}^{n+1}=1-s / 2 \quad \text { and } \quad \forall 1 \leq i<n, \quad d_{i, n+1}^{n+1}=d_{i n}^{n}+1-s .
$$


This is indeed a definition of a metric as long as $0<s \leq \min \left\{1,2 \min _{1 \leq i<n} d_{i, n}^{n}\right\}$ (this fact follows from a simple case analysis).

We are left to check condition (b). Fix $1 \leq i<j<k \leq n$. If $k \neq n$, then

$$
\begin{aligned}
& d_{i, j}^{n+1}+d_{j, k}^{n+1}- d_{i, k}^{n+1}-\eta_{n} \\
&= d_{i, j}^{n}+d_{j, k}^{n}-d_{i, k}^{n}-\eta_{n} \\
& \geq 2 d_{j, k}^{n}\left[\delta^{-1}\left(\frac{d_{i, k}^{n}+d_{k, n}^{n}-d_{i, n}^{n}}{\min \left\{d_{i, k}^{n}, d_{k, n}^{n}\right\}}\right)+\delta^{-1}\left(\frac{d_{j, k}^{n}+d_{k, n}^{n}-d_{j, n}^{n}}{\min \left\{d_{j, k}^{n}, d_{k, n}^{n}\right\}}\right)\right] \\
& \geq 2 d_{j, k}^{n}\left[\delta^{-1}\left(\frac{d_{i, k}^{n}+\left(d_{k, n}^{n}+1-s\right)-\left(d_{i, n}^{n}+1-s\right)}{\min \left\{d_{i, k}^{n}, d_{k, n}^{n}+1-s\right\}}\right)\right. \\
&\left.+\delta^{-1}\left(\frac{d_{j, k}^{n}+\left(d_{k, n}^{n}+1-s\right)-\left(d_{j, n}^{n}+1-s\right)}{\min \left\{d_{j, k}^{n}, d_{k, n}^{n}+1-s\right\}}\right)\right] \\
&=2 d_{j, k}^{n+1}\left[\delta^{-1}\left(\frac{d_{i, k}^{n+1}+d_{k, n+1}^{n+1}-d_{i, n+1}^{n+1}}{\min \left\{d_{i, k}^{n+1}, d_{k, n+1}^{n+1}\right\}}\right)\right. \\
&\left.+\delta^{-1}\left(\frac{d_{j, k}^{n+1}+d_{k, n+1}^{n+1}-d_{j, n+1}^{n+1}}{\min \left\{d_{j, k}^{n+1}, d_{k, n+1}^{n+1}\right\}}\right)\right] .
\end{aligned}
$$

It remains to check (b) for the quadruple $\{i, j, n, n+1\}$. Condition (b) for $M_{n}$ implies that

$$
d_{i j}^{n+1}+d_{j n}^{n+1}-d_{i n}^{n+1} \geq \eta_{n}
$$

On the other hand,

$$
\begin{array}{r}
2 d_{j, n}^{n+1}\left[\delta^{-1}\left(\frac{d_{i, n}^{n+1}+d_{n, n+1}^{n+1}-d_{i, n+1}^{n+1}}{\min \left\{d_{i, n}^{n+1}, d_{n, n+1}^{n+1}\right\}}\right)+\delta^{-1}\left(\frac{d_{j, n}^{n+1}+d_{n, n+1}^{n+1}-d_{j, n+1}^{n+1}}{\min \left\{d_{j, n}^{n+1}, d_{n, n+1}^{n+1}\right\}}\right)\right] \\
=2 d_{j, n}^{n}\left[\delta^{-1}\left(\frac{s / 2}{\min \left\{d_{i, n}^{n}, 1-s / 2\right\}}\right)+\delta^{-1}\left(\frac{s / 2}{\min \left\{d_{j, n}^{n}, 1-s / 2\right\}}\right)\right],
\end{array}
$$

so that condition (b) will hold when $s$ is small enough such that the quantity above is at most $\eta_{n} / 2$ and with $\eta_{n+1}=\eta_{n} / 2$.

Corollary 11. For all $1<p<\infty, R_{p}(n)=3$ for $n \geq 3$.

We end this section with a simple lower bound for the isometric Ramsey problem for graphs. We do not know the asymptotically tight bound in this setting.

Proposition 12. Let $G$ be an unweighted graph of order $n$. Then there is a set of $\Omega(\sqrt{\log n / \log \log n})$ vertices in $G$ whose metric embeds isometrically into $\ell_{2}$.

Proof. Let $\Delta$ be the diameter of $G$. The shortest path between two diameterically far vertices is isometrically embeddable in $\ell_{2}$. On the other hand, the Bourgain et al. theorem 
[4] yields, for every $0<\varepsilon<1$, a subset $N \subset V$ which is $(1+\varepsilon)$ embeddable in Hilbert space and $|N|=\Omega((\varepsilon / \log (2 / \varepsilon)) \log n)$. When $\varepsilon=1 / 2 \Delta$, such an embedding is an isometry. Hence we can always extract a subset of $V$ which is isometrically embeddable in $\ell_{2}$ with cardinality

$$
\Omega\left(\max \left\{\Delta, \frac{\log n}{\Delta \log \Delta}\right\}\right)=\Omega\left(\sqrt{\frac{\log n}{\log \log n}}\right),
$$

as claimed.

\section{Acknowledgment}

The authors express their gratitude to Guy Kindler for some helpful discussions.

\section{References}

1. Y. Bartal, N. Linial, M. Mendel, and A. Naor. On metric Ramsey-type phenomena. Ann. of Math., to appear.

2. J. Bergh and J. Löfström. Interpolation Spaces. An Introduction. Grundlehren der Mathematischen Wissenschaften, No. 223. Springer-Verlag, Berlin, 1976.

3. B. Bollobás. Random Graphs, second edition. Cambridge University Press, Cambridge, 2001.

4. J. Bourgain, T. Figiel, and V. Milman. On Hilbertian subsets of finite metric spaces. Israel J. Math., 55(2):147-152, 1986.

5. W. Brinkman and M. Charikar. On the impossibility of dimension reduction in $\ell_{1}$. In Proceedings of the 44th Annual Symposium on Foundations of Computer Science, pages 514-523, 2003.

6. M. M. Deza and M. Laurent. Geometry of Cuts and Metrics. Springer-Verlag, Berlin, 1997.

7. A. Dvoretzky. Some results on convex bodies and Banach spaces. In Proceedings of the International Symposium on Linear Spaces (Jerusalem, 1960), pages 123-160. Jerusalem Academic Press, Jerusalem, 1961.

8. P. Enflo. On a problem of Smirnov. Ark. Mat., 8:107-109, 1969.

9. P. Erdös. Some remarks on the theory of graphs. Bull. Amer. Math. Soc., 53:292-294, 1947.

10. W. B. Johnson and J. Lindenstrauss. Extensions of Lipschitz mappings into a Hilbert space. In Conference in Modern Analysis and Probability (New Haven, Conn., 1982), pages 189-206. American Mathematical Society, Providence, RI, 1984.

11. J. R. Lee and A. Naor. Embedding the diamond graph in $L_{p}$ and dimension reduction in $L_{1}$. Geom. Functional Anal., to appear.

12. C. J. Lennard, A. M. Tonge, and A. Weston. Generalized roundness and negative type. Michigan Math. J., 44(1):37-45, 1997.

13. C. Lund and M. Yannakakis. The approximation of maximum subgraph problems. In Proceedings of ICALP, volume 700 of Lecture Notes in Computer Science, pages 40-51. Springer-Verlag, Berlin, 1993.

14. J. H. Wells and L. R. Williams. Embeddings and Extensions in Analysis. Ergebnisse der Mathematik und ihrer Grenzgebiete, Band 84. Springer-Verlag, New York, 1975.

Received December 17, 2002, and in revised form December 21, 2003. Online publication July 2, 2004. 